\title{
Dayside magnetopause Pe 3 and Pc 5 ULF waves observed by the GEOTAIL Satellite
}

\author{
Tohru Sakurai ${ }^{1}$, Yutaka Tonegawa ${ }^{1}$, Takuya Kitagawa ${ }^{1}$, Kiyofumi Yumoto $^{2}$, Tatsundo Yamamoto ${ }^{3}$, \\ Susumu Kokubun ${ }^{4}$, Toshifumi Mukai ${ }^{3}$, and Koichiro Tsuruda ${ }^{3}$ \\ ${ }^{1}$ Department of Aeronautics and Astronautics, School of Engineering, Tokai University, 1,117 Kitakaname, Hiratsuka, Kanagawa 259-1292, Japan \\ ${ }^{2}$ Department of Earth and Planetary Sciences, Kyushu University, 6-10-1 Hakosaki, Fukuoka 812-8581, Japan \\ ${ }^{3}$ The Institute of Space and Aeronautical Science, 3-1-1 Yoshinodai, Sagamihara, Kanagawa 229-8510, Japan \\ ${ }^{4}$ Solar Terrestrial Environmental Laboratory of Nagoya University, Toyokawa 442-8507, Japan
}

(Received January 26, 1998; Revised June 3, 1999; Accepted August 2, 1999)

\begin{abstract}
This study is intended to reveal the oscillation and propagation characteristics of ULF waves observed near the dayside magnetopause. A comparative study of such ULF wave phenomena is made by using the data obtained from the successive skimmings of the dayside magnetopause by the GEOTAIL Satellite performed on three successive days of 9, 18, and 27 December 1994. The instrumentations of GEOTAIL are good for such a task, since the satellite simultaneously measured the magnetic and electric fields, and low energy plasma data. Based the observed data, we studied the dynamic spectral characteristics of both magnetic and electric fields. The results revealed that the dominant ULF waves appearing in the dynamic spectra were Pc 3 and Pc 5 oscillations. The Pc 3 oscillations were observed during a limited local time around noon without an extension to the dawn and dusk flanks, and dominantly appeared at a frequency of $\sim 25 \mathrm{mHz}$ in the azimuthal component of the magnetic field. This frequency component showed clear resonant oscillation characteristics. In addition to this, a fast mode earthward propagation characteristic was also recognized. The Poynting flux of the Pc 3 signals showed that the energy flux was about 10 $\mathrm{nJ} / \mathrm{m}^{2} \mathrm{~s}$ on average, the strongest being along the magnetic field line. Pc 5 oscillations showed another dominant spectral power which appeared in the radial component of the electric field, suggesting that the resonance oscillations were well established. However, the Pc 5 oscillations in the subsolar region did not show any earthward propagation characteristics.
\end{abstract}

\section{Introduction}

Continuous Pc 3 (10-45 s) pulsations are typical dayside active pulsations which have been established through ground observations (Saito, 1969).

The geosynchronous satellite ATS 6 has provided information concerning the wave characteristics of the magnetic pulsations observed near the equator at $L=6.6$. Arthur et al. (1977) and Arthur and McPherron (1977) discovered the occurrence of distributions of Pc 3 pulsations at local time, i.e., azimuthally polarized oscillations occurring primarily in the local morning with an occurrence peak during the midmorning hours ( $\sim 1000$ LT), but the most common frequency at $35 \mathrm{mHz}$, and radially polarized oscillations showing no discernible local time-frequency dependence with frequencies higher than the dominant frequency, most of these events having significant compressional components. Arthur and McPherron (1981) also found the occurrence distribution of Pc 4 pulsations at local time, i.e., a primary peak showing in the evening at $18 \mathrm{LT}$ and a secondary peak occurring during the morning hours (at 0600 LT), but no predominance near noon. However, a strong noon peak has been reported by Cummings et al. (1975). They discovered it by studying the

Copy right (C) The Society of Geomagnetism and Earth, Planetary and Space Sciences (SGEPSS); The Seismological Society of Japan; The Volcanological Society of Japan; The Geodetic Society of Japan; The Japanese Society for Planetary Sciences. magnetic field data obtained by the ATS 1 satellite. Such a difference in local time dependence of the occurrence of Pc 4 pulsations might be attributed to the satellite location with respect to the magnetic equator. Takahashi and McPherron (1982, 1984) employed dynamic spectral analysis to determine harmonic resonance structures of Pc 3 waves observed by ATS 6.

Beyond $L=6.6$, Heppner et al. (1970) used HEOS 1 data (Hedgecock, 1976) and found that compressional Pc 3 pulsations were observed over a wide range of $L$ values extending to $12 \mathrm{Re}$ in the duskside magnetosphere.

Recent extensive studies of the pulsations have been made using data from the Active Magnetosphere Particle Tracer Explorer, Charge Composition Explorer (AMPTE/CCE) spacecraft, whose orbit was almost on the equator, extending to $8.8 \mathrm{Re}$ in the outer magnetosphere. Many studies have been carried out, particularly concerning harmonically structured resonances (Engebretson et al., 1986; Zanetti et al., 1987; Anderson et al., 1990) and radially polarized pulsations near the equator (Engebretson et al., 1988; Takahashi et al., 1990).

The nodal structure of standing oscillations in the magnetosphere at an $L$ value extending from 2.8 to 9.7 and at the magnetic latitude from the equator to $48^{\circ}$ has been examined in detail by Cahill et al. (1986) using amplitude variations of 
the magnetic and electric fields and plasma data observed by DE 1 satellite. The examination focused on Pc 3, 4 and 5 pulsations. The nodal structures of these pulsations have been clearly identified by both calculations of relative amplitude of the magnetic and electric fields and the phase difference between them. The results are found to be in agreement with the model calculations predicted by Cummings et al. (1969).

Statistical surveys for the spatial distribution of occurrences of ULF waves, including Pc3, Pc4 and Pc5, have been performed by Cao et al. (1994) based on the magnetic field data from the ISEE 1 spacecraft. Their results showed that Pc 3 waves most frequently occur just outside the synchronous orbit and are approximately centered on the local noon. Pc 4 waves show a similar distribution, but with its peak further out. Pc 5 waves have a high occurrence rate at the two flanks of the magnetosphere. Peaks in spectra obtained near the magnetopause are less clearly defined than those deeper in the magnetosphere.

The GEOTAIL satellite also provided a lot of magnetic and electric field data, and plasma data. ULF pulsation study has been promoted by using these physical data. Dayside ULF waves including Pc 3 and Pc 5 pulsations have been examined by Nakamura et al. (1994), Takahashi et al. (1994) and Tonegawa et al. (1997). Takahashi et al. (1994) mainly examined $\mathrm{Pc} 3$ pulsations especially for the identification of the fast mode wave, while Nakamura et al. (1994) contributed to refining a standing oscillation characteristic of Pc 5 pulsations. Their studies clarified the wave mode characteristics excited near the low latitude dayside magnetopause. Tonegawa et al. (1997) examined an interesting phenomenon of Pc 5 with a peculiar wave form superimposed with higher frequency impulses, which were frequently observed at the duskside magnetopause. These Pc 5 waves must be considered as being important when we consider how the wave energy transmits into the magnetosphere across the magnetopause.

The present study is intended to study ULF wave oscillation and propagation characteristics near the magnetopause, especially in the low latitude boundary layer of the dayside magnetopause, where the GEOTAIL satellite has traversed most frequently since December 1994. The examination begins with a general survey of both the magnetic and electric field variations. This is described in Section 3. The dynamic spectral characteristics for the ULF signals observed in both the magnetic and electric fields throughout the satellite trajectory are presented in Section 4, where the spectral characteristics of a variety of ULF waves seen in the dynamic spectra are discussed. Section 5 deals with the wave mode characteristics for typical Pc 3 and Pc 5 wave trains observed mainly near the subsolar magnetopause. Section 6 summarizes the important results examined in the previous sections and discusses several important characteristics concering wave propagation in relation to simultaneous observations of the plasma properties by the satellite.

\section{Data Used and Analysis Procedure}

Data used in the present study are based on magnetic (MGF) and electric field (EFD) data and low energy plasma (LEP) data obtained by the GEOTAIL satellite when the satellite skimmed the dayside magnetopause on three successive days of 9, 18, and 27 December 1994. Each trajectory of the satellite is illustrated in Fig. 1 in a X-Y plane of GSE coodinates with three different dotted curves. The perigee of the satellite was about 8 Earth radii $(\mathrm{Re})$ around noon and the apogee was $-48 \mathrm{Re}$ in the midnight meridian. Average locations of the dayside magnetopause and the bow shock are represented by a solid and dotted curve based on the models given by Sibeck et al. (1991) and Fairfield (1971), respectively. An arrow marked on the trajectories shows the 0 UT of each day, which is located in the dawnside flank each day.

It is important to note that the trajectory of the satellite was slightly different in each sector of local time, although the satellite took almost a similar trajectory. In the morning sector, the trajectory of 9 December was closest to the average magnetopause, while the trajectories of the other two days, 18 and 27 December, were more inside than that of 9 December. However, in the afternoon sector, the trajectories were taken as a reversal relationship to each other. The trajectory of 27 December was outermost and took the outbound path across the magnetopause around 17 MLT. The trajectory of 9 December was located innermost. The case of 18 December was intermediate. In the subsolar region, the satellite took almost the same trajectory and in detail, however, there was a slight difference between them. The trajectory was outermost for 9 December and innermost on 18 December. The case of 27 December was intermediate. Therefore, it is expected that the most prominant characteristics of ULF waves excited at different local times along the magnetopause can be examined in detail.

The instruments and data processings have been described

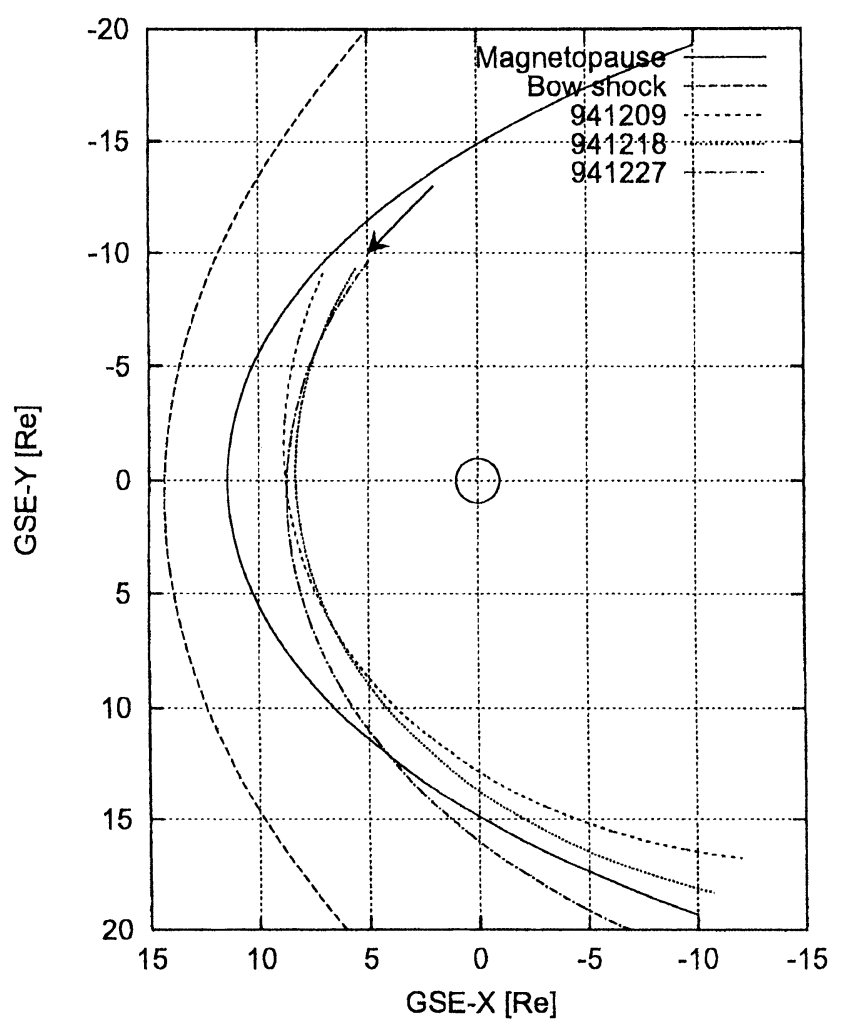

Fig. 1. The GEOTAIL satellite trajectories of 9, 18 and 27 December 1994 projected in the $X-Y$ plane of the GSE coordinates, when the satellite skimmed the dayside magnetopause. 
in detail for the magnetic field, electric field, and low energy plasma data by Kokubun et al. (1994), Tsuruda et al. (1994) and Mukai et al. (1994), respectively.

In the present analysis, we used $3 \mathrm{sec}$ average magnetic and electric field data. Each component of both fields is transformed from satellite coordinates to mean-magnetic field coordinates, from which the mean-magnetic field is determined along with the three component magnetic field data for each 10 min interval. The mean-field-aligned coordinates is used in order to present the oscillation characteristics with respect to the mean-magnetic-field direction, where $\boldsymbol{e}_{z}$ is in the direction of the mean magnetic field; $\boldsymbol{e}_{y}$ is parallel to $\boldsymbol{e}_{z} \times \boldsymbol{r}$, where $\boldsymbol{r}$ is a position vector of the satellite relative to the center of the Earth, and $\boldsymbol{e}_{x}$ (radial) is given by $\boldsymbol{e}_{x}=\boldsymbol{e}_{y} \times \boldsymbol{e}_{z}$. The parallel electric field $E_{z}$ is zero by assumption.

The dynamic spectra are calculated for each transformed component of the magnetic and electric fields by using an autoregressive (AR) method which calculates the spectral parameters by fitting an autoregressive model to a time series. Application of the AR method to ULF wave analysis has been made by many authors (e.g., Iwabuchi et al., 1979; Sato and Kokubun, 1980; Glangeaud, 1982; Tonegawa and Fukunishi, 1984; Tonegawa et al., 1984; Kato et al., 1996). Their results indicate that the AR method gives power spectra much smoother than those calculated by the classical spectral methods, i.e., Blackman-Turkey or fast Fourier transform (FFT). The smoothness of the spectral power is particularly important for obtaining accurate coherency and phase relationships between the two different field components.

Frequency resolution is important in obtaining a clear understanding of dynamic spectral behavior of the dominant power, especially in the low frequency range of Pc 5. The AR method gives us a logarithmically equal frequency resolution within the frequency range (Tonegawa et al., 1984). In the present calculation of the dynamic spectra, we employed two types of display of dynamic spectra. The first is a 24hour display for obtaining the global behavior of ULF waves throughout one day, and the other is a dynamic spectrum for inspecting the detail behavior of the Pc 3 frequency range for a specific time interval. In the latter case, we used a linear frequency scale, which clearly presented the behavior of the dynamic spectra in the Pc 3 frequency range.

In order to make the most of the advantages of the AR method, the order of AR process should be reasonably determined. The minimum final prediction error (FPE) criterion (Akaike, 1971) is generally available to find the best order for it. The minimum FPE criterion, however, fails to give a reasonable result, or a minimum value of FPE does not exist when the power spectrum has very sharp spectral peaks, and also when the signal to ratio is low. Akaike and Nakagawa (1972) have empirically indicated that the order of the AR process should be less than the value in the range of $2 \sqrt{N} / m-3 \sqrt{N} / m$, where $N$ is the number of data points and $m$ is the dimensionality of the power. This criterion has been improved upon by Kato et al. (1996) by considering that the most appropriate order of the AR process depends sensitively on the dominant frequency of the spectra.

In this study, the calculation of dynamic spectra is performed using different time windows. In order to have a 24-hour display of the dynamic spectra, a time window of
$20 \mathrm{~min}$ is taken for each spectral calculation by successively shifting $10 \mathrm{~min}$ data. However, for inspecting details of Pc 3 spectra for a specific interval, the data for a time window of $10 \mathrm{~min}$ are calculated by successively shifting $1 \mathrm{~min}$ data. The $90 \%$ data are then overlapped for each spectrum. The spectra are obtained with a smooth display. In both analyses, the background power is subtracted by fitting a second-order polynomial to the original spectrum in order to emphasize the spectral peaks, and then the ratio of the spectral peak power to the background power is calculated. The power ratio is displayed with a gray code, which covers $10 \mathrm{~dB}$ in the dynamic range of the power ratio.

\section{General Characteristics of Magnetic and Elec- tric Field Variations of 18 December 1994}

As shown in Fig. 1, the Geotail satellite skimmed the magnetopause from the dawnside to the duskside magnetopause with a path through the subsolar region, where the satellite observed the various types of magnetic and electric field oscillations characterizing each region of the magnetopause.

Before examining the details, it seems to be very instructive to see the general field variations observed along the trajectory. In the upper panel of Fig. 2, three component magnetic field variations are illustrated in the dipole coordinates, $V, D$ and $H$, where $V$ is radially outward, $D$ is azimuthally eastward, and $H$ directs antiparallel to the dipole axis.

First we note that the $V$ and $H$ components behaved in a similar way, i.e., when the satellite passed the dawnside magnetopause, the $V$ and $H$ components, and also the magnitude of the field increased gradually and attained a maximal intensity around the subsolar region at 06:00 UT (12.8 MLT) and then the field intensity gradually decreased as the satellite passed by the postnoon sector. When the satellite entered the duskside magnetopause, the field further weakened and large amplitude impulsive variations appeared there.

One of the interesting field variations was the large amplitude compressional oscillations, which appeared in the $V$ component magnetic field, $B_{v}$ and its intensity, $B_{t}$ in the duskside flank from $\sim 10$ UT (16.2 MLT) on, where the field intensity decreased from $\sim 50 \mathrm{nT}$ at $\sim 10 \mathrm{UT}(16.2 \mathrm{MLT})$ to $\sim 10 \mathrm{nT}$ at $\sim 16$ UT (18.5 MLT). The peak-to-peak amplitude of the $B_{v}$ variations attained $\sim 20 \mathrm{nT}$, showing almost the same magnitude as the field intensity. Hereafter, the impulsive variations became dominant and superposed on the compressional oscillations.

The $D$ component variations exhibited a clear signature which was different from those of the other two components, i.e., first, large amplitude impulsive variations were observed in the dawnside magnetopause and then the field became steady and reached almost zero, and changed its polarity at $\sim 05$ UT (11.8 MLT) from negative to positive, and continued its positive value with large amplitude field variations until the end of the orbit.

The electric field variations are shown in the bottom two panels, $E_{v}$ and $E_{d}$ components, respectively. The electric field was measured primarily with two components, i.e., parallel and perpendicular components to the satellite spin axis. These two components of the satellite coordinates are transformed into two components of the dipole coordinates under 

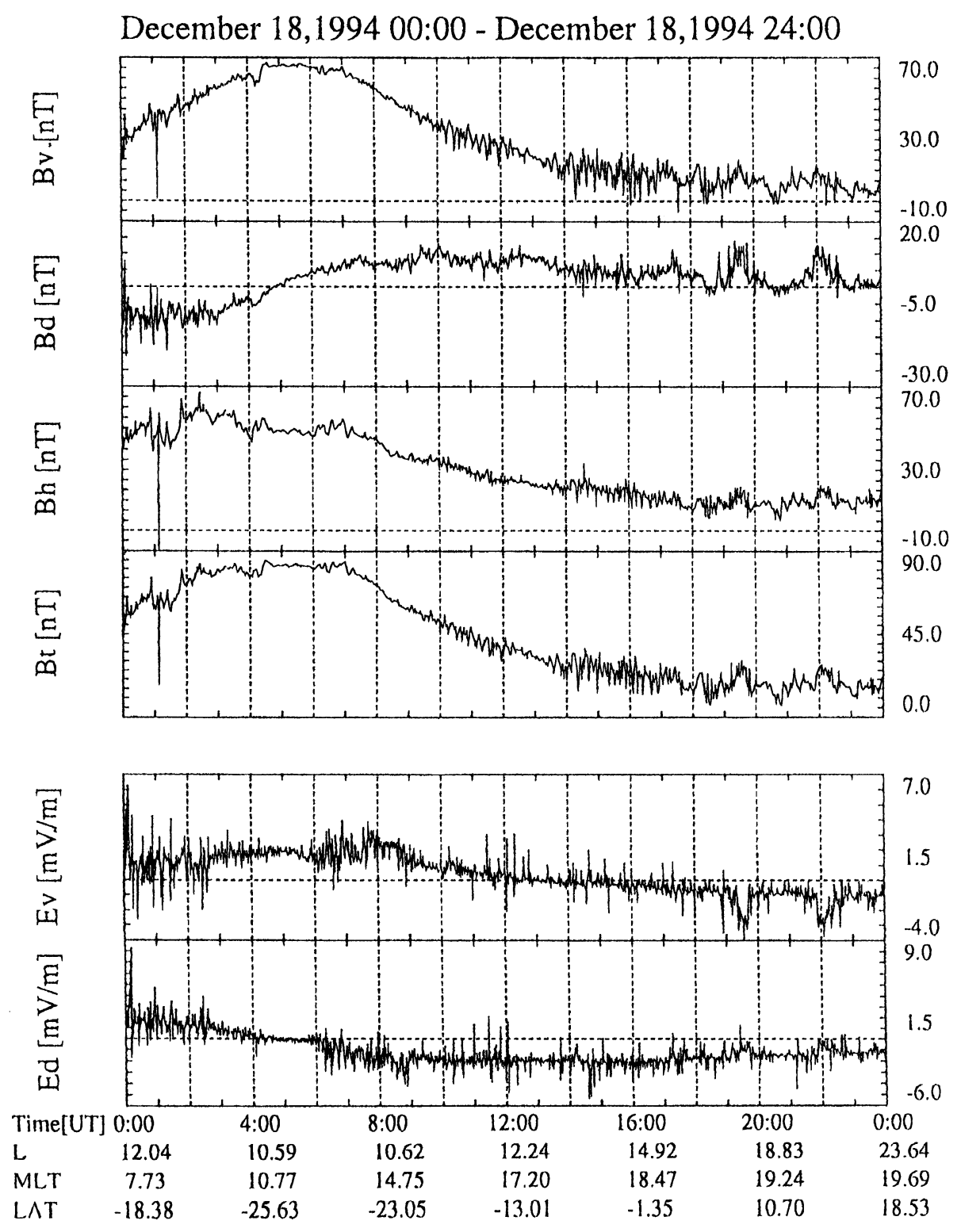

Fig. 2. Typical magnetic field and electric field variations observed on 18 December 1994. In the upper panels, from top to bottom, are shown three components of the magnetic field in a dipole coordinate, radial component $\left(B_{v}\right)$, azimuthal component $\left(B_{d}\right)$, and anti-parallel dipole-axis component $\left(B_{h}\right)$, and the field magnitude $\left(B_{t}\right)$, respectively. In the lower panels, two components of the electric field, radial component $\left(E_{v}\right)$, and azimuthal component $\left(E_{d}\right)$, respectively are shown.

the assumption of $\boldsymbol{E} \cdot \boldsymbol{B}=0$.

The electric field variations were different from the magnetic field variations. Large amplitude impulsive oscillations were apparent throughout the day and were especially significant in a radial component of the electric field, $E_{v}$, in the dawn and dusk flanks. The impulsive oscillations were intensive from the dawnside flank. Then they became quiescent and changed their characteristics to a regular oscillation, which continued through the subsolar region and activated from $\sim 06$ UT for about two hours. The oscillations during this interval appeared as Pc 5 ULF signals, which will be clearly understood from the dynamic spectra presented in the next section.

The impulsive oscillations were again observed in the duskside flank when the satellite passed through the low latitude boundary layer (LLBL). The compressional Pc 5 signals appeared in the $E_{d}$ component superposed on the impulsive oscillations.

Thus, from the above descriptions, it can be summarized that three types of field variations are identified, i.e., one is the large amplitude impulsive oscillations that occurred in the dawn and duskside flanks. The others are the oscillations in the frequency range of $\mathrm{Pc} 5$ (less than $10 \mathrm{mHz}$ ), i.e., one is the transverse Pc 5 oscillations that evidently appeared in the $E_{v}$ component and the other one is the compressional oscillations appearing only in the magnetic field. However, there is one more interesting and important oscillation signature which is not evidently recognized in this figure because of its small amplitude. This will, however, appear as a clear Pc 3 ULF signal in the dynamic spectrum obtained during a period when the satellite passed through the subsolar magnetopause. The details will be described in the next section 


\section{Dynamic Spectrum of Magnetic Field and Electric Field (Mean-Magnetic-Field Coordinates)}

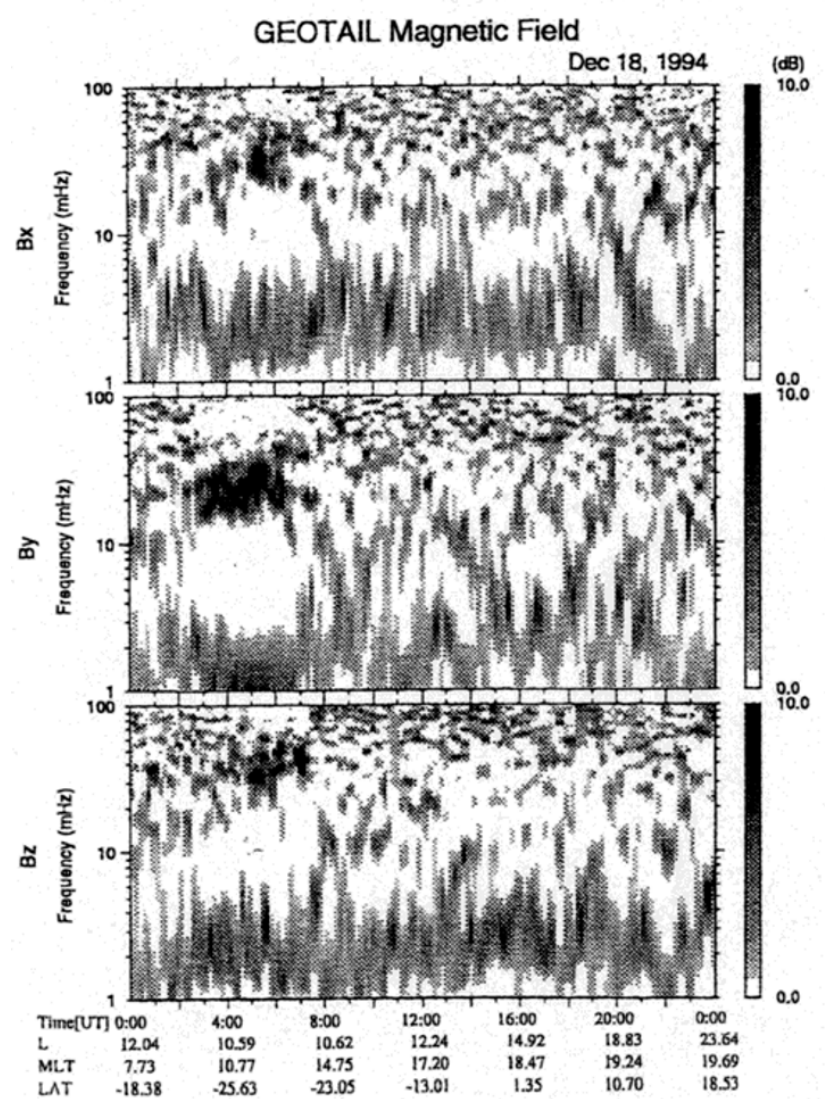

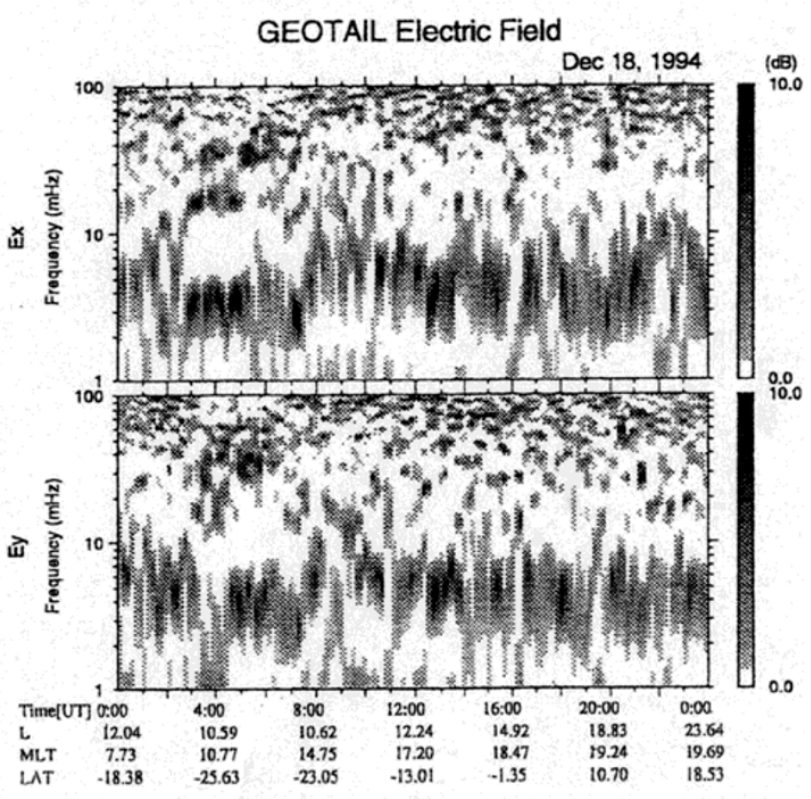

Fig. 3. Dynamic spectra of three components of the magnetic field (left-hand panels), and electric field (right-hand panels), from top to bottom, three components, $x, y$, and $z$ in a mean magnetic-field coordinate, are presented, respectively, for 18 December 1994. Note that a lumped spectral power was clearly recognized in the Pc 3 frequency band of the $B_{y}$ component of the magnetic field for about 5 hours from 0300 to 0800 UT when the satellite passed through the subsolar magnetopause. Similar Pc 3 activity enhancement is also recognized in the $B_{x}$ and $B_{z}$ components at a frequency slightly higher than that of the $B_{y}$ component. The Pc 5 spectra are clearly recognized in the radial component of the electric field, $E_{x}$

by using the dynamic spectra.

\section{Dynamic Spectral Characteristics of the Mag- netic and Electric Field Variations}

\subsection{Case study of 18 December 1994}

A typical example of the dynamic spectra obtained on 18 December 1994 is presented in Fig. 3 for each component of the magnetic and electric fields in the left and right panels, respectively. In this section, the dynamic spectra of both field components are calculated based on the data obtained by using the mean-magnetic field coordinates.

An inspection of the figure produces two important points. One is the remarkable activity of the Pc 3 signals in a frequency range from 20 to $30 \mathrm{mHz}$ and the other is the activity of Pc 5 in a frequency of less than $10 \mathrm{mHz}$.

A remarkable activity of the Pc 3 signals is depicted as a lumped enhancement of the spectral power in the azimuthal component of the magnetic field, $B_{y}$, which is exhibited in a limited time interval (or a spatial extent) only when the satellite passed through the subsolar magnetopause. This result is, however, consistent with the previous satellite study by Cao et al. (1994) based on the ISEE 1 satellite magnetic field data. The Pc 3 specral power was dominant near the subsolar region.

The Pc 3 spectra also appear in both the radial and compressional components of the magnetic field, $B_{x}$ and $B_{z}$, in a little higher frequency range. However, they are less evident than that of the $B_{y}$ component of the magnetic field. In the electric field, the Pc 3 spectra are less evident than those of the magnetic field.

The other point to be noted from the dynamic spectra is the Pc 5 spectra, which are clearly recognized in the electric field spectra rather than those of the magnetic field. Comparing the spectra of the two components, $E_{x}$ and $E_{y}$, of the electric field, the Pc 5 is enhanced in the $E_{x}$ component rather than the $E_{y}$ component. The Pc 5 spectra are also recognized around local noon, which appear with the Pc 3 spectra in the $B_{y}$ component, although the Pc 5 spectra also appear over a wide range of local times extending from the dawn to dusk flanks.

In order to examine the detailed spectral structures of Pc 3 for both the magnetic and electric fields, enlarged displays of the spectra are shown in Fig. 4, which is represented for a limited interval of two hours from 04 to 06 UT around the subsolar region. In order to extract only the Pc 3 signals, we calculated the spectra using the time-derivative data of the 


\section{Dynamic Spectrum of Magnetic Field and Electric Field (Mean-Magnetic-Field Coordinates)}
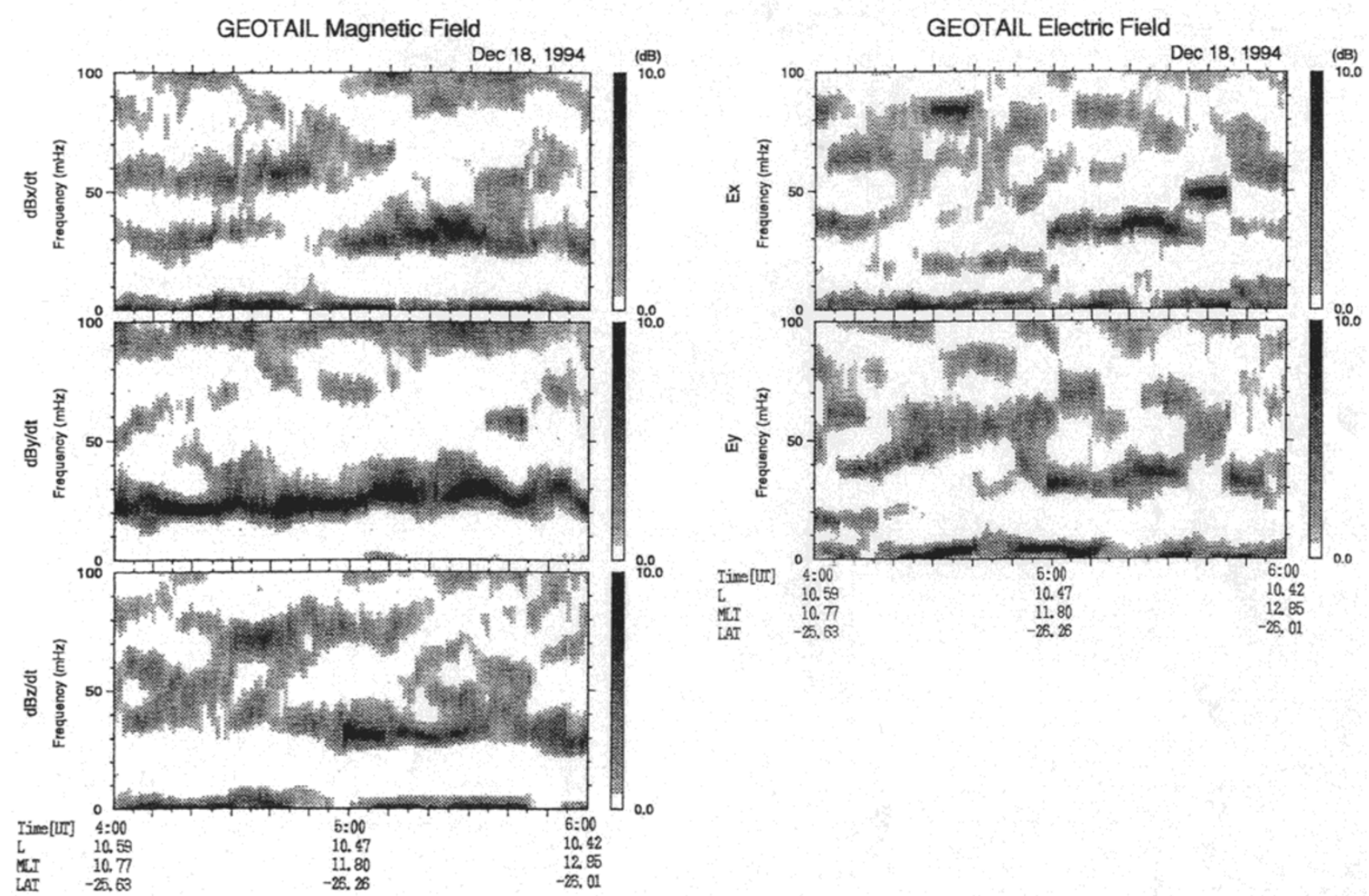

Fig. 4. Enlarged presentation for each component of the magnetic (left) and electric (right) fields during 2 hours from 0400 to 0600 UT, which corresponds to the satellite location near the subsolar magnetopause. A clear narrow-band horizontal spectral structure can be seen in the $B_{y}$ component of the magnetic field in the Pc 3 frequency range. Similar spectral bands cannot be identified in any other field components.

magnetic field. Each spectrum is calculated for a time interval of 10 min successively shifting $1 \mathrm{~min}$ data. Therefore, each dynamic spectrum is given by overlapping $90 \%$ of the data which enables us to obtain a very smooth spectrum.

In the figure, the enhancement of the spectral power of Pc 3 is clearly found in the azimuthal component of the magnetic field, $B_{y}$ and is characterized by a horizontal structure centered at a frequency of about $25 \mathrm{mHz}$. However, the frequency of the spectral band varies slightly and the spectral power is activated intermittently with a 10 to 20 min interval. Similar activity changes of Pc 3 have been found in the outer magnetosphere by Chi and Russell (1998) from surveying the ISEE 1 satellite data.

From the dynamic spectral study, it is concluded that we can recognize two dominant spectral activities from the Pc 3 and Pc 5 signals. They appear in the different field components, suggesting that each spectrum specifies different oscillation and propagation modes. More detailed examinations of the oscillation and propagation modes will be performed in Section 5.

\subsection{Case study of 9 December 1994}

The dynamic spectra of the three components of the magnetic and the two components of the electric fields of 9 December 1994 are not shown here. However, we can summa- rize briefly the spectral characteristics as follows:

1. The spectrum of Pc 3 was not clearly apparent on 9 December. The spectral power was rather weaker than that of 18 December. This may be due to the satellite location with respect to the magnetopause, which was outermost, close to the magnetopause, in the prenoon sector on this day.

2. On the other hand, the Pc 5 spectrum was clearly identified especially in the dusk flank. The activity was recognized over a wide range extending from $L=12$ to $L=16$. The strong spectral power appeared at a frequency of $\sim 3$ $\mathrm{mHz}$ in the radial component, $E_{x}$, of the electric field, suggesting that Pc 5 is established as a clear resonant oscillation there.

Another interesting fact for Pc 5 oscillations concerns the oscillating wave form. The Pc 5 oscillations described above showed a peculiar wave form superposed with high frequency oscillations at the positive extremes of the oscillations, which have been reported by Tonegawa et al. (1997).

3. Compressional oscillations were also recognized in this Pc 5 event. The causal relation between the transverse and compressional oscillations seems to be very inter- 
esting. However, in this case, the resonant power in the transverse component was superior to the compressional power. This observed fact might be due to the satellite's location relative to the source region of the Pc 5 pulsations. In this case, the satellite passed along the inside of the magnetosphere rather than the path of 18 December. The 18 December path was close to the magnetopause. Thus, the compressional power was found to be superior to that of the transverse resonant oscillations.

\subsection{Case study of 27 December 1994}

The dynamic spectra of the magnetic and electric fields of 27 December are also not shown here. The spectral characteristics are summarized as follows:

1. The most significant spectral feature was that of the Pc 3 spectra which were dominant in the azimuthal component of the magnetic field, $B_{y}$. The enhanced spectra were also recognized as a lumped enhancement similar to that of 18 December. However, this enhancement was only in the $B_{y}$ component and did not appear in the other components of the magnetic field and also in any component of the electric field.

2. Any spectral enhancement of Pc 5 could not be identified. This may be due to the satellite path with respect to the magnetopause. On this day, the satellite was outermost, close to the magnetopause, crossing the magnetopause at 15 MLT, and after that it was in the magnetosheath.

Summarizing the observed spectra of the three successive magnetopause skimmings on 9, 18 and 27 December, the most common feature is an enhancement of the spectral power of the Pc 3 ULF signal in a limited region near the subsolar magnetopause. Away from the subsolar region there was no enhancement of the Pc 3 spectra. This is a very significant feature in the appearance of $\mathrm{Pc} 3$ signals. Contrary to the spectral behavior of the Pc 3 signals, the Pc 5 signals were activated over a wide range of the local time from the dawn to dusk magnetopause. The activity was different between the Pc 3 and Pc 5 signals, which is very important in cosidering a generation and energy transmission mechanism of these ULF signals.

\section{Wave Mode Characteristics of Pc 3 and Pc 5 ULF Signals}

The examination of the cross-phase relationship between $B_{y}$ and $E_{x}$ components is discussed in the first step. The calculated cross-phase spectrum between them is shown in Fig. 5. From top to bottom, the panels show the auto-power spectrum of each field component, cross-power spectrum of both field components, coherence, and the phase angle between them, respectively. A dominant power with a high degree of coherence appears at the frequency $\sim 24 \mathrm{mHz}$. The cross-phase angle is about $90^{\circ}$ at this frequency. A positive $90^{\circ}$ phase angle means that the phase of the $B_{y}$ component advances $90^{\circ}$ with respect to that of the $E_{x}$ component. This result suggests that the Poynting flux of this transverse wave propagates along the magnetic field-line, suggesting that a standing oscillation is established at this frequency.

The other two subsidiary spectral peaks also stand at fre-
Evidence of Field-Line Resonance Phase Relation Between the By And the Ex Components

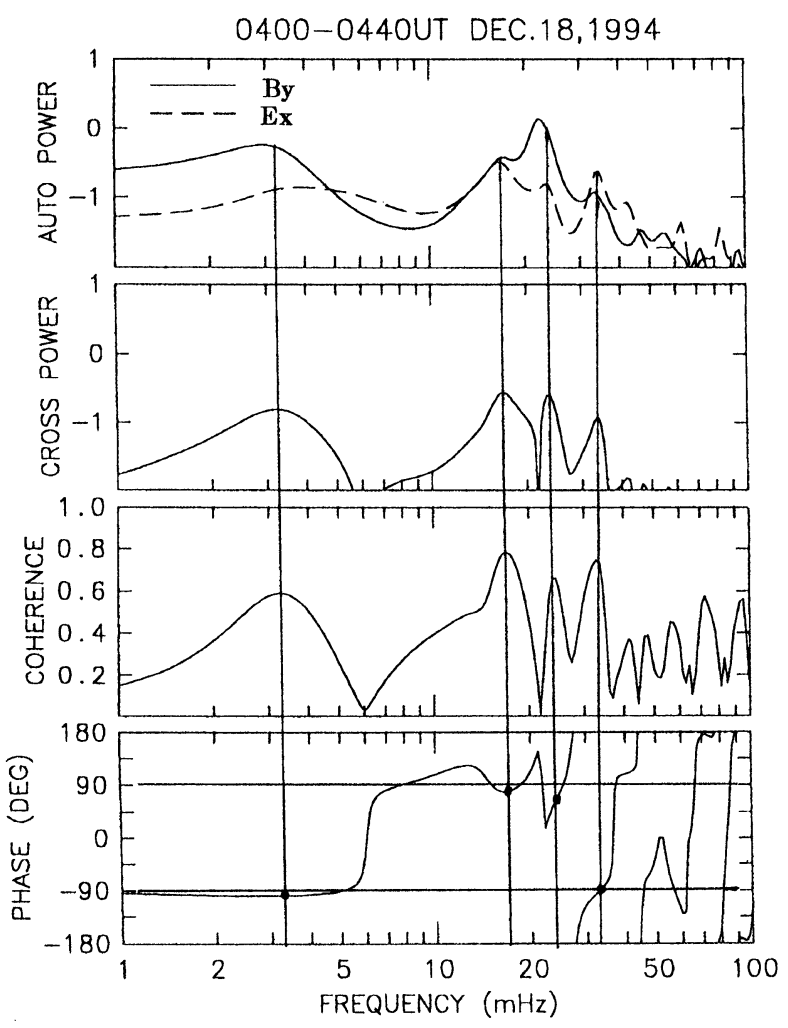

Fig. 5. A cross-spectrum for the resonant oscillation is illustrated using both components of the azimuthal component, $B_{y}$, of the magnetic field and the radial component of the electric field, $E_{x}$, for a 40 min interval from 0400 to 0440 UT. Auto- and cross-power, coherence and phase angle are shown in the panels from top to bottom, respectively. Spectral peaks with a high coherence stand at four frequencies of 3, 18, 22 and 35 $\mathrm{mHz}$. The corresponding phase angles are almost the same and exhibit a $90^{\circ}$ phase shift. This result indicates that these spectral peaks are due to resonant field-line oscillations.

quencies of $\sim 18$ and $\sim 32 \mathrm{mHz}$ at both sides of the dominant spectral peak. These subsidiary spectral peaks belong to the frequencies of Pc 3. The phase angles corresponding to these frequencies are about $90^{\circ}$, indicating that the oscillation shows a resonant field-line oscillation.

While a high degree of coherence stands at the frequency of $\sim 3 \mathrm{mHz}$, where a clear $90^{\circ}$ phase lag of the azimuthal component of the magnetic field, $B_{y}$ with respect to the radial component of the electric field, $E_{x}$ can be recognized. This is also an indication of the standing oscillation.

A cross-phase relation for the compressional oscillation is also examined using the magnetic field, $B_{z}$, and the azimuthal component of the electric field, $E_{y}$, and is shown in Fig. 6. A dominant cross-spectral peak occurs at the frequency of Pc 3 . A high degree of coherence stands at a frequency of about 38 $\mathrm{mHz}$ with other subsidiary spectral peaks which belong to the frequency of Pc 3. The spectral power of these peaks is less intense than those of the transverse component. The phase angle between the magnetic field, $B_{z}$, and the electric field, $E_{y}$, at these spectral peaks is about $180^{\circ}$. This $180^{\circ}$ phase 
Phase Relation Between $\mathrm{Bz}$ and Ey components

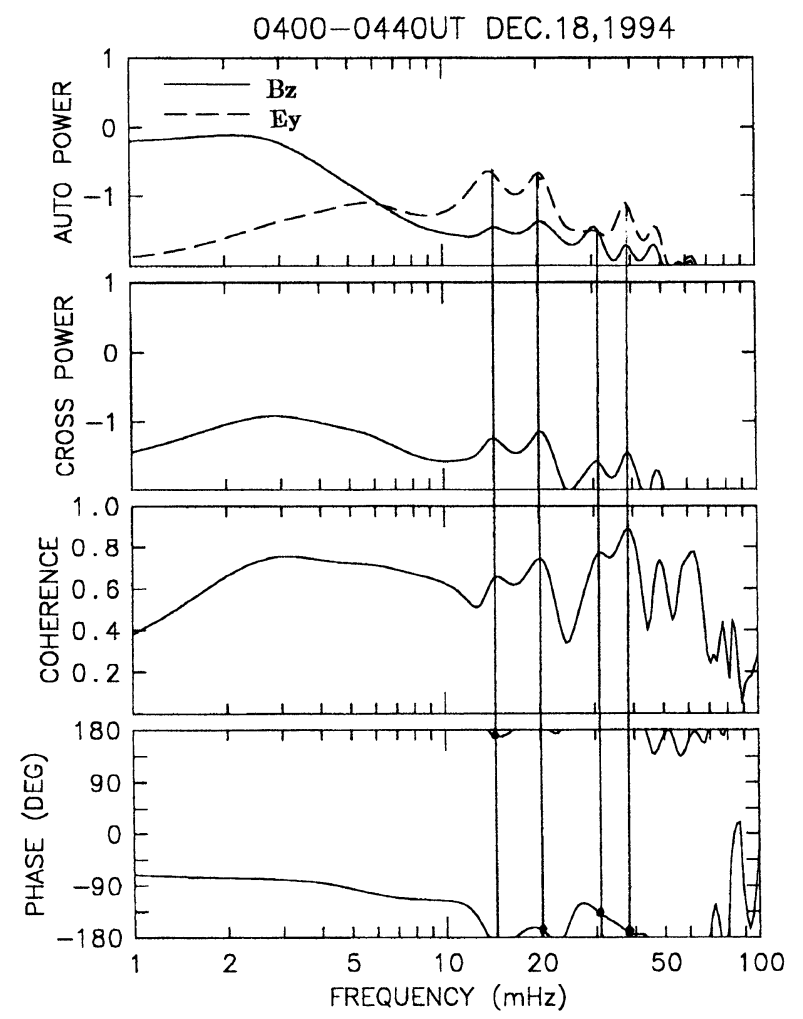

Fig. 6. A cross-spectrum for the compressional oscillation for the same interval is shown. The format is the same as in Fig. 5 except for the field components $B_{z}$ and $E_{y}$. Note that high coherent oscillations occur at frequencies of $15,20,30,40,50$, and $60 \mathrm{mHz}$, almost similar to the frequencies seen in Fig. 5, and the cross-phase angles at these frequencies are almost a $180^{\circ}$ phase shift, suggesting that the compressional energy at these frequencies radially propagate earthward.

angle suggests that the Poynting flux of the wave energy propagates earthward, since the phase angle zero corresponds to sunward propagation.

In this case, however, Pc 5 shows no discernible earthward propagation characteristics.

In conclusion, the auto- and cross-spectral analyses carried out in this section indicate that similar resonant oscillation characteristics can be identified in both the Pc 3 and Pc 5 signals, although the compressional component of the Pc 3 shows an earthward propagation, while the Pc 5 does not indicate such an earthward propagation characteristic.

\section{Summary and Discussion}

In the previous sections, we examined the dynamic spectral characteristics using magnetic and electric field data, which were measured in the three successive subsolar magnetopause skimmings of the GEOTAIL satellite during the days of 9, 18 and 27 December 1994. Dominant spectra were observed in the Pc 3 and Pc 5 ULF signals. We can summarize our observed facts as follows:

(1) The most significant fact obtained from the present dynamic spectral analysis is that Pc 3 and Pc 5 spectra were observed to be dominant.
(2) The Pc 3 spectra were observed with the azimuthal component of the magnetic field, but only in a limited longitude near the subsolar magnetopause. In addition, the Pc 3 oscillations simultaneously showed both resonant and compressional oscillation characteristics.

(3) The Pc 5 spectrum was another dominant spectrum, which was recognized in the electric field over the wide range of the local time, suggesting that a fundamental mode oscillation was clearly observed by GEOTAIL, since the satellite traversed near the magnetic equator.

(4) The cross-phase examinations clarified that there was a clear $90^{\circ}$ phase lag between the $B_{y}$ and $E_{x}$ components at the frequencies of both Pc 3 and Pc 5. This fact means that the Poynting energy of these oscillations directs towards the magnetic field-line, suggesting that a field-line resonance is established for both categories of ULF waves.

(5) A similar cross-phase analysis using the compressional component of the magnetic field, $B_{z}$, and the azimuthal component of the electric field, $E_{y}$, showed highly coherent spectral peaks at the frequency of Pc 3, where the phase angle shows $180^{\circ}$ at the spectral peak, suggesting that the compressional energy propagates radially earthward. Such an earthward propagation is not clearly recognized in the Pc 5 oscillations.

(6) The resonant oscillations of Pc 5 were clearly identified, even near the subsolar magnetopause on 18 December.

(7) Usually, the resonant oscillations of Pc 5 were observed in both the flanks and, close to the magnetopause boundary of the flank region, the compressional oscillations, however, appeared to be dominant. Therefore, the resonant and/or nonresonant characteristics of the Pc 5 oscillations seem to depend on the satellite location with respect to the magnetopause boundary.

As pointed out in item (1), the most prominent feature in the present study is the lumped appearance of the activity of the Pc 3 power near the subsolar magnetopause. Anderson et al. (1990) and Anderson and Engebretson (1995) have reported the appearance of Pc 3 oscillations for a fairly wide range of the daytime in the outer magnetosphere from the AMPTE/CCE satellite. However, Cao et al. (1994) have stated from their spectral analysis based on the data of the magnetic field from the ISEE 1 satellite, that the Pc 3 pulsations were observed to be dominant near the subsolar region.

The result obtained in the present study reveals that the local time extent of the Pc 3 activity is rather limited near the subsolar region. This is consistent with the result reported by Cao et al. (1994).

From our present examination, we suggest that the spatial extent should have a constraint on the model of energy transmission which is more likely to be the subsolar magnetopause for Pc 3 waves.

The Poynting fluxes of the Pc 3 oscillations are calculated for a time interval from 0400 to 0430 UT on 18 December 1994. Figure 7 illustrates a band-pass filtered amplitude versus time variation of the three components of the magnetic 


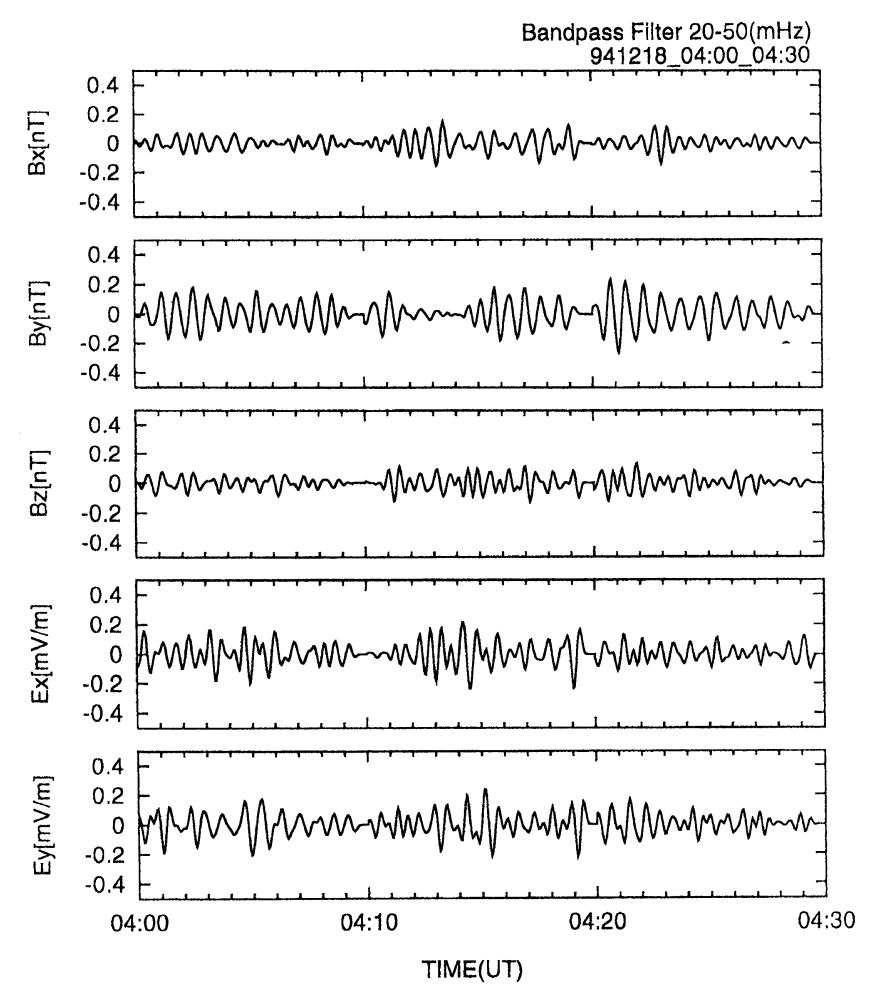

Fig. 7. In the panels from top to bottom, are the band-pass filtered Pc 3 amplitude versus time variations of the three components of the magnetic field, $E_{x}, E_{y}$ and $E_{z}$, and the two components of the electric field, $E_{x}$ and $E_{y}$ in the mean-field aligned coordinates during $30 \mathrm{~min}$ from 0400 to 0430 UT on 18 December 1994, respectively.

field and two components of the electric field with a bandpass filtered frequency width of $30 \mathrm{mHz}$ from 20 to $50 \mathrm{mHz}$, which covers the dominant spectral frequency of the Pc 3 spectra shown in Fig. 4. The average peak-to-peak amplitude of each component of the magnetic field is about $0.2 \mathrm{nT}$ or less, except for the large amplitude oscillations of the $B_{y}$ component, in which the amplitude is, on average $0.3 \mathrm{nT}$. For the electric field oscillations, the average amplitude is $0.3 \mathrm{mV} / \mathrm{m}$ for both components.

By using these oscillating field components, we have tried to calculate the Poynting flux $\boldsymbol{S}$ where $\boldsymbol{S}=\boldsymbol{E} \times \boldsymbol{B} / \mu_{0}$. The time variations of the three components of the Poynting flux, $\boldsymbol{S}_{x}, \boldsymbol{S}_{y}$ and $\boldsymbol{S}_{z}$, are shown in Fig. 8 in the top to bottom panels, respectively. It is very interesting to note that the radial component of the Poynting flux is predominantly nagative, meaning that the wave energy propagates predominantly earthward. The azimuthal component is also interesting in that the energy propagates almost toward the afternoon side. In the direction along the magnetic field-line, the energy repeatedly bounces back and forth along the magnetic field-line, thus, establishing the resonance oscillations.

The absolute value of the energy flux amounts to about $10 \mathrm{~nJ} / \mathrm{m}^{2} \mathrm{~s}$ on average in each component. If we consider the energy flux across the dayside magnetopause, assuming a longitudinal extent of about 6 hours around the subsolar point and \pm 10 degrees in the latitudinal extent at a radial distance of $10 \mathrm{Re}$ from the center of the Earth, we can calculate the total energy flux into the inner magnetosphere. The result is proved to be $10^{12} \mathrm{~W}$, which is two orders of magnitude

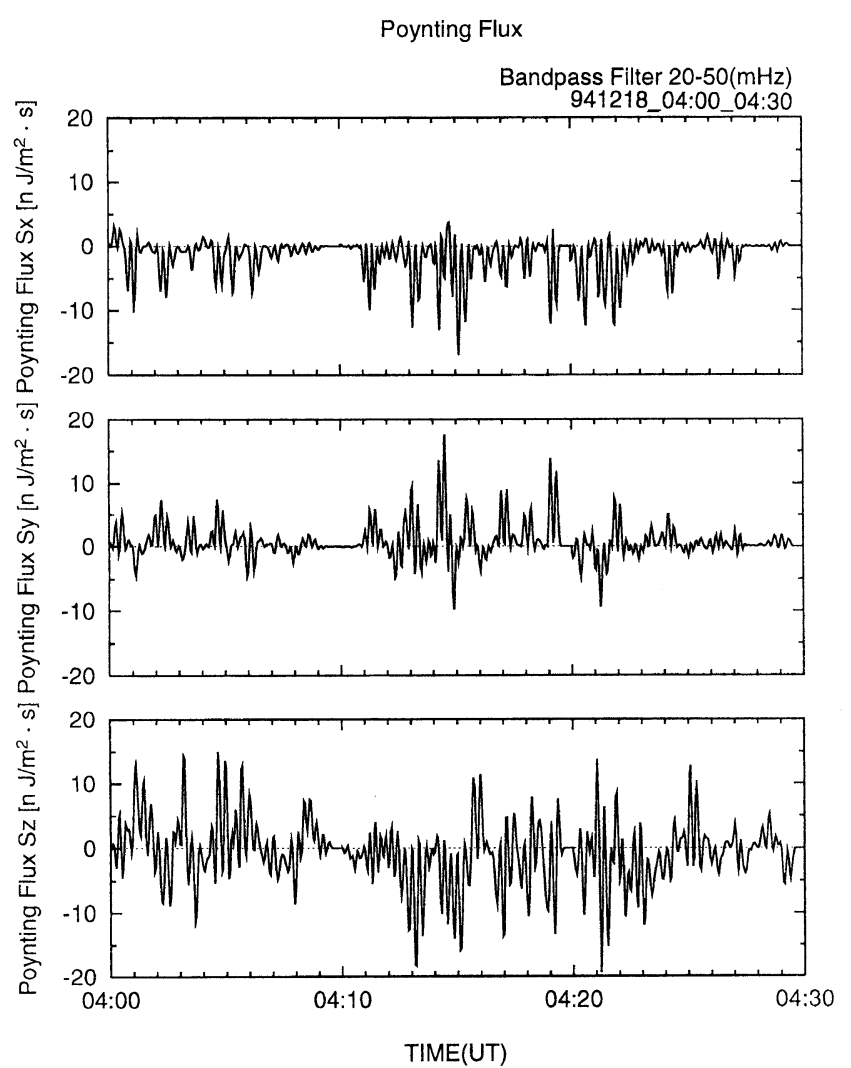

Fig. 8. The Poynting fluxes in the mean-field aligned coordinates for the Pc 3 event of Fig. 7

less than the hourly value of the magnetospheric substorm energy, $10^{14} \mathrm{~W}$ (Greenwald and Walker, 1980). However, it is important to remenber that the Pc 3 wave energy is continuously fed into the magnetosphere from the magnetosheath side.

Similar examinations of the Poynting flux of the Pc 3-4 waves in the outer magnetosphere have been reported quite recently by Chi and Russell (1998). Therefore, both results seem to be important in considering the energy budget of the magnetosphere.

As for the propagation of the Pc 3 waves, we should recall, however, some of the evidence previously reported by Song et al. $(1992,1993)$ based on a study using ISEE satellite data. They showed that a slow mode hydromagnetic wave was dominant near the subsolar magnetosheath. Energy transmission from the magnetosheath into the magnetosphere might be expected to be mainly slow mode hydromagnetic waves, and then the transmitted energy might excite the eigenmode of the local field-line resonant oscillations.

This scenario can be verified in our present study. The phase relationships examined in Section 4 on both transverse and compressional modes, suggest that both modes can be identified almost simultaneously at the same frequency near the subsolar magnetopause. Therefore, it is expected that the field-line resonance might come into operation because of the transmitted compressional wave energy at the inside of the magnetopause.

It is also very interesting to check which mode, fast or slow, corresponds to the compressional wave discussed above. We 
Table 1. Location of the satellite.

\begin{tabular}{ccccccc}
\hline & \multicolumn{2}{c}{$L$-value } & \multicolumn{2}{c}{ MLT } & \multicolumn{2}{c}{ mag. lat. } \\
\hline Date & $4: 00(\mathrm{UT})$ & $5: 00(\mathrm{UT})$ & $4: 00(\mathrm{UT})$ & $5: 00(\mathrm{UT})$ & $4: 00(\mathrm{UT})$ & $5: 00(\mathrm{UT})$ \\
\hline Dec. 09 & 11.19 & 11.01 & 10.99 & 11.91 & -25.13 & -26.05 \\
Dec. 18 & 10.59 & 10.52 & 10.77 & 11.28 & -25.63 & -26.05 \\
Dec. 27 & 11.02 & 10.92 & 10.57 & 11.38 & -25.02 & -26.14 \\
\hline
\end{tabular}

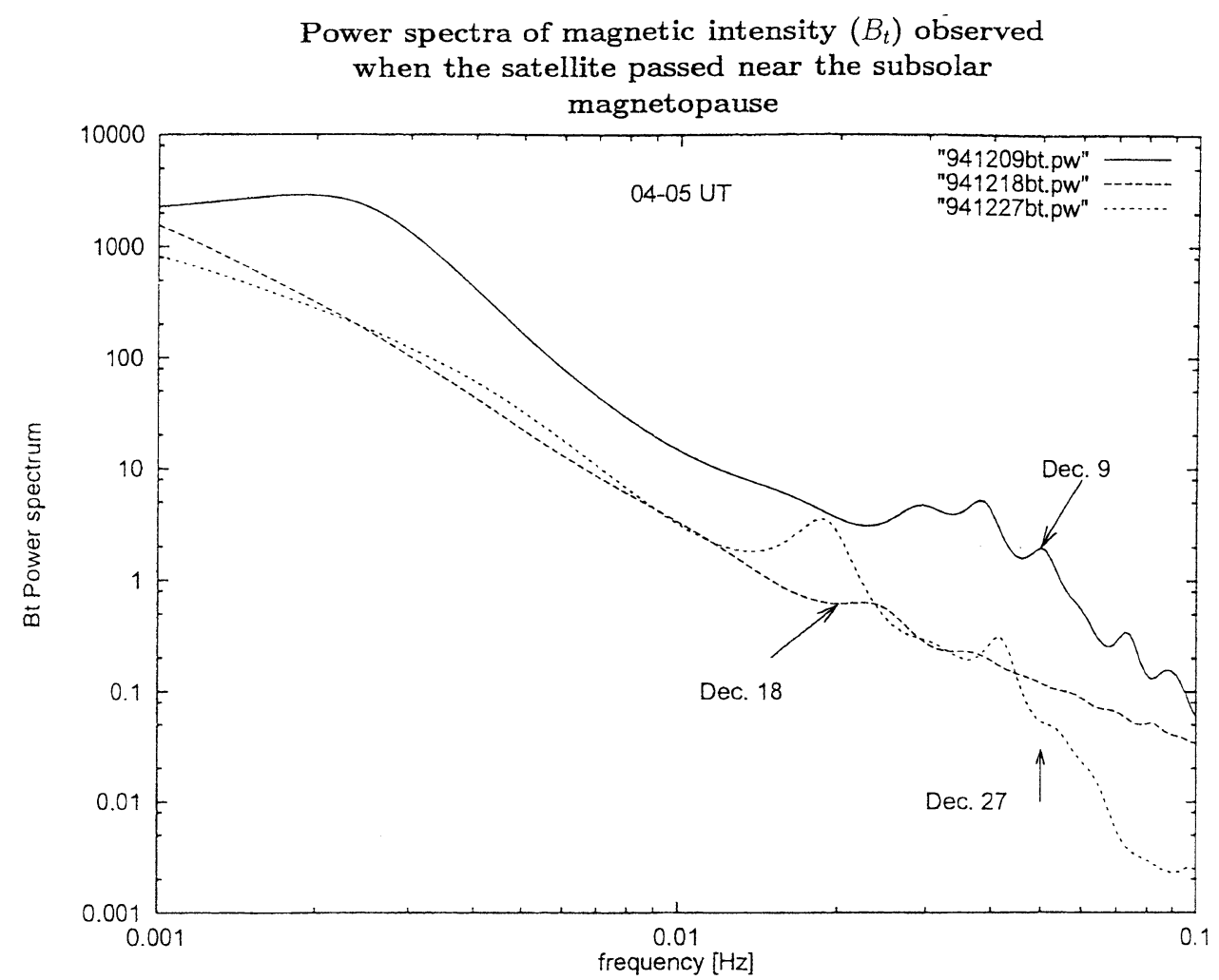

Fig. 9. Spectral power of the magnetic field intensity, $B_{t}$, for one hour from 0400 to 0500 UT on the three days of 9 , 18 , and 27 December 1994 . The time interval corresponds to the satellite passes through the subsolar magnetopause. Note that the power is largest on 9 December, while during 18 December, the power is weakest.

examined this point by using the data observed on 18 December. The method used in this study was developed by Takahashi et al. (1994) for an examination of the wave mode of a Pc 3 event observed on 18 December.

The result showed that the phase speed of the compressional Pc 3 oscillations was about $1700 \mathrm{~km} / \mathrm{s}$, which was more appropriate to the calculated fast mode speed of about $1900 \mathrm{~km} / \mathrm{s}$ and not to the slow mode speed of about $300 \mathrm{~km} / \mathrm{s}$. Cross-spectral analysis between the two components of the fields $B_{z}$ and $E_{y}$, showed a $180^{\circ}$ phase lag between them, indicating that the compressional wave of the Pc 3 propagates earthward as a fast mode. Thus, we can conclude that the fast mode wave is more preferable for explaining the compressional Pc 3 wave observed near the subsolar magnetopause on 18 December. This result is consistent with the result obtained by Takahashi et al. (1994).

Moreover, it is expected that the spectral power would be more intense at the outer region of the magnetopause if we could assume that the wave energy might be transmitted from the magnetosheath into the magnetosphere as suggested by Greenstadt et al. (1980, 1983) and Yumoto et al. (1984, 1985). This point can be examined by using the data of the present study. The trajectories of the three successive magnetopause skimmings of 9, 18, and 27 December are suitable for checking this point, because the trajectories were almost the same, being only slightly different each other, which is evidently shown in Fig. 1. The satellite locations are given in Table 1 with three parameters such as the $L$ value, MLT, and magnetic latitude at the times of 0400 and 0500 UT, respectively. From Table 1, it is clear that the satellite took a slightly different trajectory near the subsolar region. We made good use of this difference.

The spectral power of the magnetic field intensity, $B_{t}$, during one hour from 0400 UT to 0500 UT is given in Fig. 9 with traces of spectral power versus frequency for the three days. By comparing these three spectra, it is clear that the spectral power was strongest on 9 December, i.e., two orders of magnitude larger than that of 18 December, whose power 
Power Spectra of D-component Magnetic Field Observed When the Satellite Passed Near the

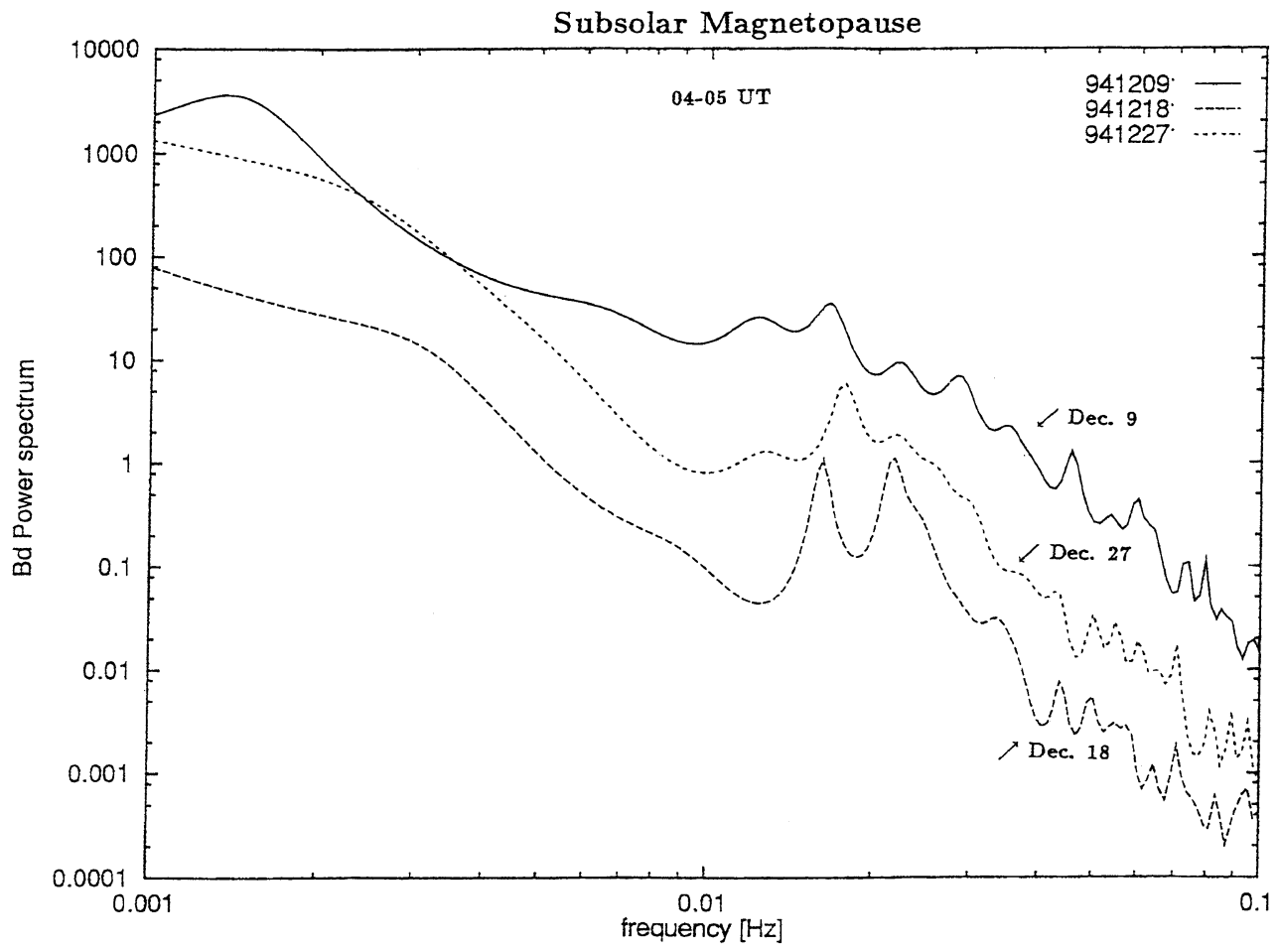

Fig. 10. Spectral power of the azimuthal component of the magnetic field, $B_{y}$. The format is the same as in Fig. 7. Spectral power was greatest on 9 December, while on 18 December, the power was weakest. However, the $Q$ value of the Pc 3 spectral peak was most pronounced on 18 December.

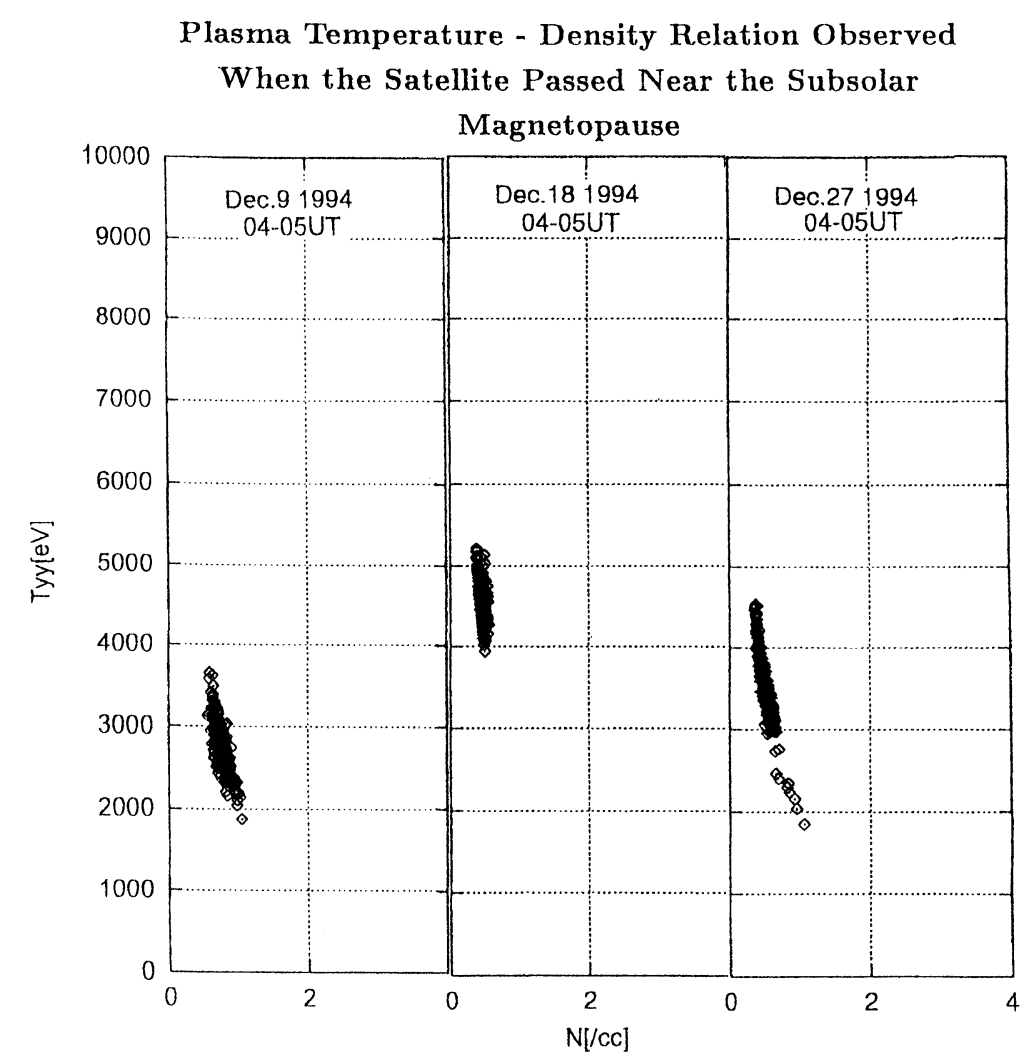

Fig. 11. Temperature versus density plots of the observed low energy plasma for the one hour interval from 0400 to 0500 UT on each day. The highest and lowest temperature plasmas were observed on 18 December and 9 December, respectively, suggesting that the satellite was located at the place closest to the subsolar magnetopause on 9 December and the highest energy plasma was oberved on 18 December, when the satellite was innermost away from the subsolar magnetopause. 


\section{Schematic illustration of ULF waves observed along the dayside magnetopause}

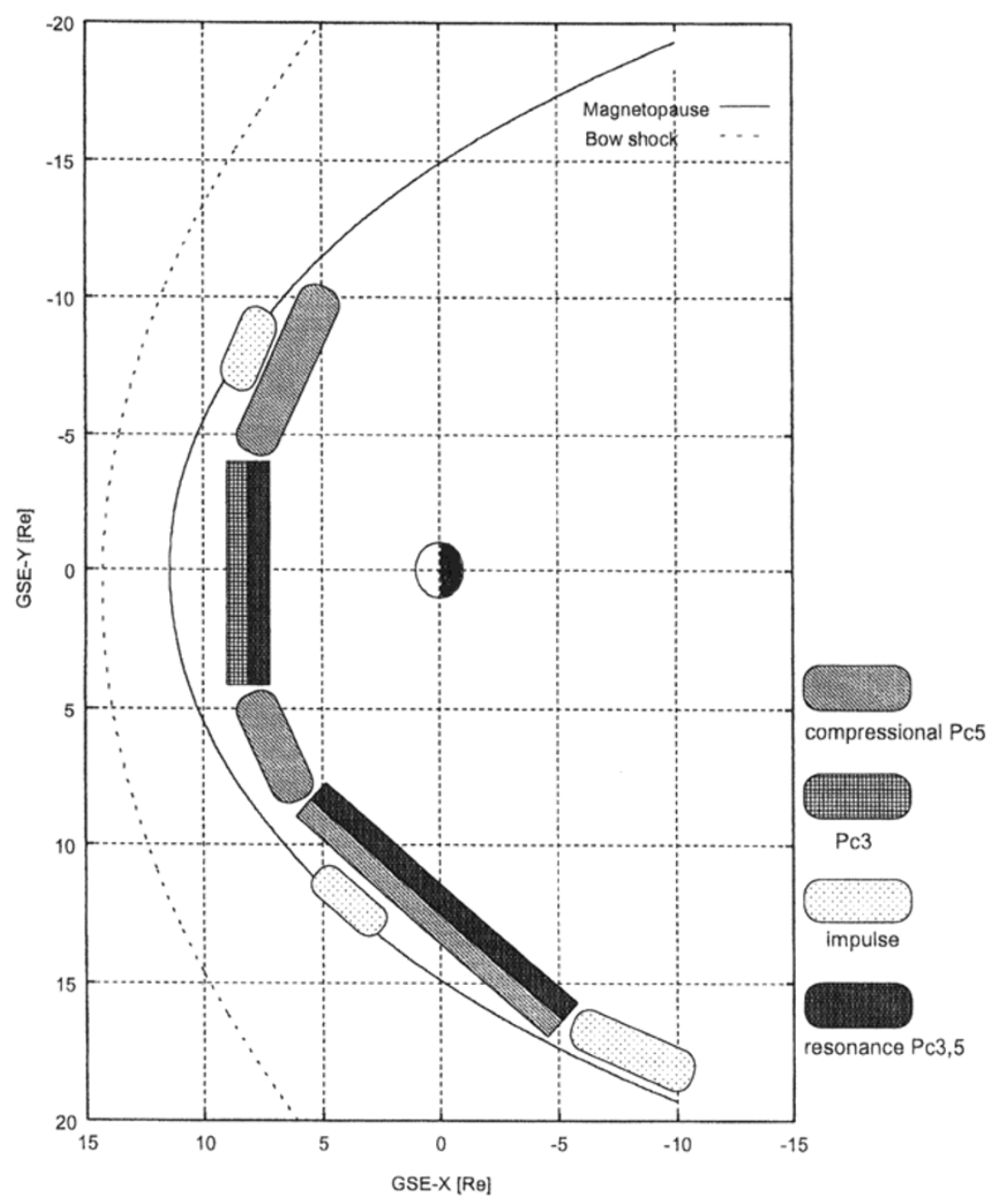

Fig. 12. A schematic illustration of the spatial occurrence distribution of ULF waves observed when the satellite skimmed the dayside magnetopause. Localized appearance of Pc 3 was most significantly observed near the subsolar magnetpause. Pc 5 oscillations were observed over a wide range of local time. Near the flanks, the compressional Pc 5 oscillations were frequently observed in association with the magnetic impulses.

was weakest. However, from a comparison among the spectra of the transverse component of the magnetic field, $B_{y}$, as shown in Fig. 10, the most pronounced spectrum with a high $Q$ value was observed at the corresponding frequency on 18 December. These results suggest that the spectral power was most prominant at the outer region close to the magnetopause, and gradually decreased toward the inside, and was weakest when the satellite stayed in the region farthest away from the magnetopause. However, the resonant oscillations are well established there, where the propagated compressional energy might be coupled most effectively to shear Alfvén waves, which make a clear resonant oscillation of the magnetic field-line.

The results seem to be reasonable when we consider that the source wave propagates into the inside of the magnetopause from the magnetosheath.

Next, we further examined the plasma property during the interval from 0400 to 0500 UT for each day. Figure 11 presents a relationship of ion temperature versus ion den- sity. The most distinct point is laid on the observed plasma temperature. On 18 December, it showed the highest temperature plasma with energy of about $4 \sim 5 \mathrm{keV}$ and the density of $N \leq 0.5 / \mathrm{cc}$, while on 9 December it gave the lowest temperature of about $2 \sim 4 \mathrm{keV}$ with the density of $N \leq 1 / \mathrm{cc}$. The lowest plasma temperature may suggest that the satellite was located in the outer region of the magnetopause. On the other hand, the highest plasma temperature means that the satellite was at the most inside of the magnetopause.

Thus, we can conclude that the spectral power was much stronger when the satellite stayed in the outer region of the magnetopause. This region corresponds to the boundary layer of the low latitude boundary layer (LLBL). The properties of plasmas discussed above are consistent with those in the LLBL reported by Le et al. $(1994,1996)$. The magnetosheath-like plasma was observed on 9 December. However, the resonance properties could be well recognized on 18 December, when the satellite stayed within the magnetospheric plasma at the inside of the magnetopause boundary. 
We can conclude from the above discussions that the difference between spectral power and resonance characteristics for the Pc 3 oscillations at each trajectory might be due to the position of the GEOTAIL satellite with respect to the magnetopause boundary.

This conclusion seems to be very important when we consider where and how source wave energy for daytime Pc 3 ULF signals penetrates into the magnetosphere, and where resonant oscillations are established.

On the other hand, from item (3), Pc 5 oscillations clearly appeared in the flanks although, on 18 December, Pc 5 oscillations were observed even near the subsolar region. The $\mathrm{Pc}$ 5 activity was, however, identified only by the electric field oscillations and there was no indication of the magnetic field variations. Previous studies used satellite magnetic field data (Heppner et al., 1970; Hedgecock, 1976; Yumoto, 1984; Anderson et al., 1990; Cao et al., 1994) but could not point out evidence of the Pc 5 oscillations near the magnetic equator. Cao et al. (1994) reported that Pc 5 oscillations were observed at the off-magnetic equator region, which might lead to a wrong understanding of the latitudinal distribution of the occurrence of Pc 5 oscillations in the outer magnetosphere.

The GEOTAIL satellite can provide electric field data, from which we can identify a lot of Pc 5 oscillations near the magnetic equator. Extremely large amplitude Pc 5 oscillations have been identified in the electric field which has recently been reported by Sakurai et al. (1999). The phenomenon is very interesting in that the Pc 5 oscillations were observed only in the dawnside flank and not in the duskside flank. The reason is not clear because theories and numerical simulations (Chen and Hasegawa, 1974; Southwood, 1974; Miura, 1984; Kivelson and Chen, 1995; Miura, 1995) so far reported, have not shown any asymmetry of occurrence probability of Pc 5 in the dawn and dusk side flanks in the outer magnetosphere.

Nosé et al. (1995) pointed out a strong dawn/dusk asymmetry of occurrence of Pc 5, which was, however, found in the inner magnetosphere by surveying the DE-1 satellite data during magnetically active periods. To explain the strong dawn/dusk asymmetry, they tried to examine the dependence of the angle between the solar wind velocity and IMF orientation in the ecliptic plane, and found that there was some evidence showing that the angle might control the Pc 5 occurrence, although only when the solar wind velocity is small. In the case of a high solar wind velocity, the causal relationship remains unclear.

We can summarize our result in Fig. 12, where the spatial distribution of the Pc 3 and Pc 5 pulsations obtained in this study is presented. Pc 3 oscillations were observed only near the subsolar region, and Pc 5 oscillations were mainly observed in the dawn- and dusk-side magnetopause. Their oscillations were observed in both the oscillation modes, the resonance, and compressional oscillations. Strong resonant oscillations were observed at the inside of the magnetopause. The other significant oscillations were the impulsive oscillations, which were mainly observed when the satellite crossed the magnetopause.

Acknowledgments. We would like to express our sincere thanks to all members of the GEOTAIL project team. The key parameter data of the Wind satellite were provided by the NASA/GSFC data processing team. The Editor thanks T. Yeoman and another referee for their assistance in evaluating this paper.

\section{References}

Akaike, H., Autoregressive model fitting for control, Ann. Inst. Stat. Math., 23, 163-180, 1971

Akaike, H. and T. Nakagawa, Statistical Analysis and Control of Dynamic System, 189 pp., Saiense-sha, Tokyo, 1972 (in Japanese).

Anderson, B. J. and M. J. Engebretson, Relative intensity of toroidal and compressional Pc3-4 wave power in the dayside magnetosphere, J. Geophys. Res., 100(A6), 9591-9603, 1995.

Anderson, B. J., M. J. Engebretson, S. P. Rounds, L. J. Zanetti, and T. A. Potemra, A statistical study of Pc 3-5 pulsations observed by the AMPTE/CCE magnetic fields experiment, 1. Occurrence distributions, J. Geophys. Res., 95(A7), 10,495-10,524, 1990.

Arthur, C. W. and R. L. McPherron, Interplanetary magnetic field conditions associated with synchronous orbit observations of Pc 3 magnetic pulsations, J. Geophys. Res., 82(32), 5138-5142, 1977.

Arthur, C. W. and R. L. McPherron, The statistical character of Pc 4 magnetic pulsations at synchronous orbit, J. Geophys. Res., 86(A3), 1325-1334, 1981.

Arthur, C. W., R. L. McPherron, and W. J. Hughes, A statistical study of Pc 3 magnetic pulsations at synchronous orbit, ATS 6, J. Geophys. Res., 82(7), 1149-1157, 1977

Cahill, L. J., Jr., N. G. Lin, M. J. Engebretson, D. R. Weimer, and M. Sugiura, Electric and magnetic observations of the structure of standing waves in the magnetosphere, J. Geophys. Res., 91(A8), 8895-8908, 1986.

Cao, M., R. L. McPherron, and C. T. Russell, Statistical study of ULF wave occurrence in the dayside magnetosphere, J. Geophys. Res., 99, 87318753, 1994.

Chen, L. and A. Hasegawa, A theory of long-period magnetic pulsations, 1. Steady state excitation of field-line resonances, J. Geophys. Res., 79 1024-1032, 1974.

Chi, P. J. and C. T. Russell, Phase skipping and Poynting flux of continuous pulsations, J. Geophys. Res., 103(A12), 29,479-29,491, 1998.

Cummings, W. D., R. J. O'Sullivan, and P. J. Coleman, Standing Alfven waves in the magnetosphere, J. Geophys. Res., 74, 778-793, 1969.

Cummings, W. D., C. Coutee, D. Lyons, and W. Wiley, III, The dominant mode of standing Alfven waves at synchronous orbit, J. Geophys. Res., 80, 3705-3708, 1975 .

Engebretson, M. J., C.-I. Meng, R. L. Arnoldy, and L. J. Cahill, Jr., Pc 3 pulsations observed near the south polar cusp, J. Geophys. Res., 91(A8), 8909-8918, 1986

Engebretson, M. J., L. J. Zanetti, T. A. Potemra, D. M. Klumper, R. J. Strangeway, and M. H. Acuña, Observations of intense ULF pulsation activity near the geomagnetic equator during quiet times, J. Geophys. Res., 93, 12795-12816, 1988.

Fairfield, D. H., Average and unusual locations of the Earth's magnetopause and bow shock, J. Geophys. Res., 76, 6700-6716, 1971.

Glangeaud, F., Analysis of pulsations, Planet. Space Sci., 30, 1249-1258, 1982.

Greenstadt, E. W., R. L. McPherron, and K. Takahashi, Solar wind control of daytime midperiod geomagnetic pulsations, J. Geomag. Geoelectr., 32, 89-110, 1980 .

Greenstadt, E. W., M. M. Mellott, R. L. McPherron, C. R. Russell, H. J. Singer, and D. J. Knecht, Transfer of pulsation-related wave activity across the magnetopause: Observations of corresponding spectra by ISEE 1 and ISEE 2, Geophys. Res. Lett., 10, 659-662, 1983.

Greenwald, R. A. and A. D. M. Walker, Energetics of long period resonant hydromagnetic waves, Geophs. Res. Lett., 7, 745-748, 1980.

Hedgecock, P. C., Giant Pc5 pulsations in the outer magnetosphere: A survey of HEOS-1 data, Planet. Space Sci., 24, 921-935, 1976.

Heppner, J. P., B. G. Ledley, T. L. Skillman, and M. Sugiura, A preliminary survey of the distribution of micropulsations in the magnetosphere from OGO's-3 and 5, Ann. Geophys., 26, 709-717, 1970.

Iwabuchi, M., N. Sato, and H. Fukunishi, Application of conventional spectral analysis program (CSAP) system for magnetic pulsations observed at Mizuho and Syowa stations, Antarct. Rec., 64, 111-126, 1979.

Kato, K., Y. Tonegawa, and T. Sakurai, Study on order of autoregressive model for spectral analysis of long period magnetic pulsations, Proc. School of Engn. Tokai Univ., 36(1), 201-205, 1996 (in Japanese).

Kivelson, M. G. and S.-H. Chen, The magnetopause: Surface waves and instabilities and their possible dynamical consequences, in Geophysical Monograph 90, edited by P. Song, B. O. Sonnerup, and M. F. Tomsen, 
pp. 257-268, AGU, 1995.

Kokubun, S., T. Yamamoto, M. H. Acuña, K. Hayashi, K. Shiokawa, and H. Kawano, The GEOTAIL magnetic field experiment, J. Geomag. Geoelectr. 46, 7-22, 1994.

Le, G., C. T. Russell, and J. T. Gosling, Structure of the magnetopause for low Mach number and strongly northward interplanetary magnetic field, J. Geophys. Res., 99(A12), 23,723-23,734, 1994.

Le, G., C. T. Russell, J. T. Gosling, and M. F. Thomsen, ISEE observations of low-latitude boundary layer for northward interplanetary magnetic field: Implications for cusp reconnection, J. Geophys. Res., 101(A12), 27,23927,249, 1996.

Miura, A., Anomalous transport by magnetohydrodynamic KelvinHelmholtz instabilities in the solar wind-magnetosphere interaction, $J$. Geophys. Res., 89, 801-818, 1984.

Miura, A., Kelvin-Helmholtz instability at the magnetopause: Computer simulation, in Geophysical Monograph 90, Physics of the Magnetopause, edited by P. Song, B. O. Sonnerup, and M. F. Tomsen, pp. 285-291, AGU, 1995.

Mukai, T., S. Machida, Y. Saito, M. Hirahara, T. Terasawa, N. Kaya, T. Obara, M. Ejiri, and A. Nishida, The low energy particle(LEP) experiment, onboard the GEOTAIL Satellite, J. Geomag. Geoelectr., 46, 669$692,1994$.

Nakamura, M., H. Matsui, H. Kawano, A. Matsuoka, T. Yamamoto, K. Tsuruda, and H. Hayakawa, Pc 5 pulsations observed in the dayside magnetosphere by Geotail, Geophys. Res. Lett., 21(25), 2903-2906, 1994.

Nosé, M., T. Iyemori, M. Sugiura, and J. A. Slavin, A strong dawn/dusk asymmetry in Pc 5 pulsation occurrence observed by the DE-1 satellite, Geophys. Res. Lett., 22(15), 2053-2056, 1995.

Saito, T., Geomagnetic pulsations, Space Sci. Rev., 10, 319-412, 1969.

Sakurai, T., T. Tonegawa, T. Kitagawa, M. Nowada, A. Yamawaki, T. Mukai, T. Yamamoto, S. Kokubun, and K. Tsuruda, Double-frequency oscillations of low energy plasma in association with transverse Pc 5 oscillations, GEOTAIL satellite observations, Earth Planets Space, 51(1), 43-54, 1999.

Sato, N. and S. Kokubun, Interaction between ELF-VLF emissions and magnetic pulsations: Quasi-periodic ELF-VLF emissions associated with Pc 3-4 pulsations and their geomagnetic conjugacy, J. Geophys. Res., 85, 101-113, 1980.

Sibeck, D. G., R. E. Lopez, and E. C. Roelof, Solar wind control of the magnetopause shape, location, and motion, J. Geophys. Res., 96(A4), 5489-5495, 1991.

Song, P., C. T. Russell, and M. F. Thommsen, Slow mode transmission in the frontside magnetosheath, J. Geophys. Res., 97(A6), 8295-8305, 1992.

Song, P., C. T. Russell, R. J. Strangeway, J. R. Wygant, C. A.Cattell, R. J. Fitzenretter, and R. R. Anderson, Wave properties near the subsolar magnetoopause: Pc 3-4 energy coupling for northward interplanetary magnetic field, J. Geophys. Res., 98(A1), 187-196, 1993.

Southwood, D. J., Some features of field line resonances in the magne- topause, Planet. Space Sci., 22, 483-491, 1974.

Takahashi, K. and R. L. McPherron, Harmonic structure of Pc 3-4 pulsations, J. Geophys. Res., 87(A3), 1504-1516, 1982.

Takahashi, K. and R. L. McPherron, Standing hydromagnetic oscillations in the magnetosphere, Planet. Space Sci., 32(11), 1343-1359, 1984.

Takahashi, K., B. J. Anderson, and R. J. Strangeway, AMPTE CCE observation of Pc 3-4 pulsations at $L=2-6$, J. Geophys. Res., 95(A10), 17,179-17,186, 1990.

Takahashi, K., S. Kokubun, H. Matsuoka, K. Shiokawa, K. Yumoto, M Nakamura, H. Kawano, T. Yamamoto, A. Matsuoka, K. Tsuruda, H. Hayakawa, K. Kojima, and H. Matsumoto, Geotail observation of magnetosonic Pc 3 waves in the dayside magnetosphere, Geophys. Res. Lett., 21, 2899-2902, 1994.

Tonegawa, Y. and H. Fukunishi, Harmonic structure of Pc 3-5 magnetic pulsations observed at the Syowa -Husafell conjugate pair, J. Geophys. Res., 89, 6737-6784, 1984.

Tonegawa, Y., H. Fukunishi, T. Hirasawa, R. L. McPherron, T. Sakurai, and Y. Kato, Specral characteristics of Pc 3 and Pc 4/5 magnetic pulsation bands observed near $L=6$, J. Geophys. Res., 89(A11), 9720-9730, 1984.

Tonegawa, Y., T. Sakurai, T. Yamamoto, K. Tsuruda, and S. Kokubun, Spectral characteristics of Pc 3-5 pulsations observed by Geotail skimming the dayside magnetopause (Extended abstract), Proc. NIPR Symp. Upper Atmos. Phys., 10, 165-169, 1997.

Tsuruda, K., H. Hayakawa, M. Nakamura, T. Okada, M. Matsuoka, F. S Mozer, and R. Schmidt, Electric field measurements on the GEOTAIL Satellite, J. Geomag. Geoelectr., 46, 693-712, 1994.

Ulrych, T. J. and T. N. Bishop, Maximum entropy spectral analysis and autoregressive decomposition, Rev. Geophys. Space Phys., 13, 183-200, 1975 .

Yumoto, K., Long-period magnetic pulsations generated in the magnetosperic boundary layers, Planet. Space Sci., 32, 1205-1218, 1984.

Yumoto, K., T. Saito, B. T. Tsurutani, E. J. Smith, and S.-I. Akasofu, Relationship between the IMF magnitude and Pc 3 magnetic pulsations in the magnetosphere, J. Geophys. Res., 89, 9731-9740, 1984.

Yumoto, K., T. Saito, S.-I. Akasofu, B. T. Tsurutani, and E. J. Smith, Propagation mechanism of dayside Pc 3-4 pulsations observed at synchronous orbit and multiple ground-based stations, J. Geophys. Res., 90, 6439$6450,1985$.

Zanetti, L. J., T. A. Potemura, R. E. Erlandson, M. J. Engebretson, and M. H. Acuña, Geomagnetic field-line resonant harmonics measured by the Viking and AMPTE/CCE magnetic field experiments, Geophys. Res. Lett., 14, 427, 1987.

T. Sakurai (e-mail: sakurai@ms.u-tokai.ac.jp), Y. Tonegawa, T Kitagawa, K. Yumoto, T. Yamamoto, S. Kokubun, T. Mukai, and K. Tsuruda 